\title{
Boundary value problems for fractional differential equations with nonlocal boundary conditions
}

\author{
Rian Yan, Shurong Sun*, Ying Sun and Zhenlai Han
}

"Correspondence:

sshrong@163.com

School of Mathematical Sciences,

University of Jinan, Jinan, Shandong

250022, P.R. China

\begin{abstract}
In this paper, we establish some sufficient conditions for the existence of solutions to two classes of boundary value problems for fractional differential equations with nonlocal boundary conditions. Our goal is to establish some criteria of existence for the boundary problems with nonlocal boundary condition involving the Caputo fractional derivative, using Banach's fixed point theorem and Schaefer's fixed point theorem. Finally, we present four examples to show the importance of these results. MSC: $34 \mathrm{~A} 08 ; 34 \mathrm{~B} 10$
\end{abstract}

Keywords: fractional differential equation; boundary value problem; fixed-point theorem

\section{Introduction}

Fractional differential equations have been of increasing importance for the past decades due to their diverse applications in science and engineering such as the memory of a variety of materials, signal identification and image processing, optical systems, thermal system materials and mechanical systems, control system, etc.; see [1, 2]. Many interesting results of the existence of solutions of various classes of fractional differential equations have been obtained; see [3-17] and the references therein.

Recently, much attention has been focused on the study of the existence and multiplicity of solutions or positive solutions for boundary value problems of fractional differential equations with local boundary value problems by the use of techniques of nonlinear analysis (fixed-point theorems, Leray-Schauder theory, the upper and lower solution method, etc.); see [7-17].

On the other hand, integer-order differential equations boundary value problems with nonlocal boundary conditions arise in a variety of different areas of applied mathematics and physics. For example, heat conduction, chemical engineering, underground water flow, thermo-elasticity, and plasma physics can be reduced to nonlocal problems with integral boundary conditions. They include two, three, and nonlocal boundary value problems as special cases and have attracted the attention of Gallardo [18], Karakostas and Tsamatos [19] (also see the references therein).

In fact, there have been the same requirements for fractional differential equations. Boundary value problems for fractional-order differential equations with nonlocal boundary conditions constitute a very interesting and important class of problems [20-22].

C 2013 Yan et al: licensee Springer. This is an Open Access article distributed under the terms of the Creative Commons Attribution License (http://creativecommons.org/licenses/by/2.0), which permits unrestricted use, distribution, and reproduction in any medium, provided the original work is properly cited. 
To the best of our knowledge, we can see the fact that, although the fractional differential equation boundary value problems have been studied by some authors, very little is known in the literature on the boundary value problems with integral boundary conditions. In order to enrich the theoretical knowledge of the above, in this paper, we investigate two classes of fractional differential equation boundary value problems with integral boundary conditions.

Benchohra et al. studied the boundary value problem for the fractional differential equations with nonlocal conditions [23]

$$
\begin{aligned}
& D_{0+}^{\alpha} y(t)=f(t, y(t)), \quad t \in J=[0, T], 1<\alpha \leq 2, \\
& y(0)=g(y), \quad y(T)=y_{T},
\end{aligned}
$$

where $D_{0+}^{\alpha}$ is the Caputo fractional derivative, $f:[0, T] \times \mathbb{R} \rightarrow \mathbb{R}$ is a continuous function, $g: C(J, \mathbb{R}) \rightarrow \mathbb{R}$ is a continuous function and $y_{T} \in \mathbb{R}$.

Motivated by all the works above, in this paper we deal with the existence and uniqueness of solutions for the boundary value problem of fractional differential equations

$$
D_{0+}^{\alpha} x(t)=f(t, x(t)), \quad t \in[0, T], 1<\alpha \leq 2,
$$

subject to one of the following nonlocal boundary conditions:

$$
\begin{aligned}
& x(0)=y(x), \quad \int_{0}^{T} x(t) d t=m, \\
& x(0)=y(x), \quad x(T)=\int_{0}^{T} g(s) x(s) d s,
\end{aligned}
$$

where $D_{0+}^{\alpha}$ is the Caputo fractional derivative, $f:[0, T] \times \mathbb{R} \rightarrow \mathbb{R}$ is a $C^{2}$ continuous function, $y: C^{2}([0, T], \mathbb{R}) \rightarrow \mathbb{R}$ is a $C^{2}$ continuous functional, $g:[0, T] \rightarrow \mathbb{R}$ is a $C^{2}$ continuous function and $m \in \mathbb{R}$. Our goal is to establish some criteria of existence for boundary value problem (1.1) with nonlocal boundary condition (1.2) or (1.3) involving the Caputo fractional derivative, using Banach's fixed point theorem and Schaefer's fixed point theorem. Finally we present four examples.

\section{Preliminaries}

In this section, we introduce notations, definitions of fractional calculus and prove two lemmas before stating our main results. By $C^{2}([0, T], \mathbb{R})$ we denote the Banach space of all continuous functions from $[0, T]$ into $\mathbb{R}$ with the norm

$$
\|x\|:=\sup \{|x|: t \in[0, T]\} .
$$

Definition 2.1 [2] For a continuous function $y:(0, \infty) \rightarrow \mathbb{R}$, the Riemann-Liouville fractional integral of order $\alpha$ is defined as

$$
I_{0+}^{\alpha}=\frac{1}{\Gamma(\alpha)} \int_{0}^{t}(t-s)^{\alpha-1} y(s) d s, \quad \alpha>0,
$$

where $\Gamma$ is the gamma function. 
Definition 2.2 [2] The Caputo fractional derivative of order $\alpha$ for a continuous function $y(t)$ is defined by

$$
D_{0+}^{\alpha} y(t)=\frac{1}{\Gamma(n-\alpha)} \int_{0}^{t}(t-s)^{n-\alpha-1} y^{(n)}(s) d s, \quad \alpha>0,
$$

where $\Gamma$ is the gamma function, $n=[\alpha]+1$ and $[\alpha]$ denotes the integer of $\alpha$.

Lemma 2.1 [2] Let $\alpha>0$. Then the fractional differential equation $D_{0+}^{\alpha} h(t)=0$ has the solution $h(t)=c_{0}+c_{1} t+c_{2} t^{2}+\cdots+c_{n-1} t^{n-1}, c_{i} \in \mathbb{R}, i=0,1, \ldots, n-1$.

Lemma 2.2 [2] Let $\alpha>0$. Then $I^{\alpha} D_{0+}^{\alpha} h(t)=h(t)+c_{0}+c_{1} t+c_{2} t^{2}+\cdots+c_{n-1} t^{n-1}$ for some $c_{i} \in \mathbb{R}, i=0,1, \ldots, n-1, n=[\alpha]+1$.

Lemma 2.3 Let $1<\alpha \leq 2$ and $h(t) \in C^{2}[0, T]$. A function $x$ is a solution of the following fractional boundary value problem:

$$
\begin{aligned}
& D_{0+}^{\alpha} x(t)=h(t), \quad t \in[0, T], \\
& x(0)=y(x), \quad \int_{0}^{T} x(t) d t=m
\end{aligned}
$$

if and only if $x$ is a solution of the fractional integral equation

$$
\begin{aligned}
x(t)= & \frac{1}{\Gamma(\alpha)} \int_{0}^{t}(t-s)^{\alpha-1} h(s) d s+\frac{2 m}{T^{2}} t+\left(\frac{T-2 t}{T}\right) y(x) \\
& -\frac{2 t}{T^{2}} \cdot \frac{1}{\Gamma(\alpha+1)} \int_{0}^{T}(T-s)^{\alpha} h(s) d s .
\end{aligned}
$$

Proof By applying Lemma 2.2, we may reduce (2.1) to an equivalent integral equation

$$
x(t)=\frac{1}{\Gamma(\alpha)} \int_{0}^{t}(t-s)^{\alpha-1} h(s) d s+c_{0}+c_{1} t
$$

for some $c_{0}, c_{1} \in \mathbb{R}$. From (2.2), it follows

$$
c_{0}=y(x), \quad c_{1}=\frac{2 m}{T^{2}}-\frac{2}{T} y(x)-\frac{2}{T^{2}} \cdot \frac{1}{\Gamma(\alpha+1)} \int_{0}^{T}(T-s)^{\alpha} h(s) d s .
$$

Thus

$$
\begin{aligned}
x(t)= & \frac{1}{\Gamma(\alpha)} \int_{0}^{t}(t-s)^{\alpha-1} h(s) d s+\frac{2 m}{T^{2}} t+\left(\frac{T-2 t}{T}\right) y(x) \\
& -\frac{2 t}{T^{2}} \cdot \frac{1}{\Gamma(\alpha+1)} \int_{0}^{T}(T-s)^{\alpha} h(s) d s .
\end{aligned}
$$

Lemma 2.4 Let $1<\alpha \leq 2$ and $h(t) \in C^{2}[0, T]$ be continuous. A function $x$ is a solution of the fractional boundary value problem

$$
\begin{aligned}
& D_{0+}^{\alpha} x(t)=h(t), \quad t \in[0, T], \\
& x(0)=y(x), \quad x(T)=\int_{0}^{T} g(s) x(s) d s
\end{aligned}
$$


if and only if $x$ is a solution of the fractional integral equation

$$
\begin{aligned}
x(t)= & \frac{1}{\Gamma(\alpha)} \int_{0}^{t}(t-s)^{\alpha-1} h(s) d s+y(x)+\frac{t}{T} A(x) \\
& -\frac{t}{T \Gamma(\alpha)} \int_{0}^{T}(T-s)^{\alpha-1} h(s) d s-\frac{t}{T} y(x),
\end{aligned}
$$

where

$$
\begin{aligned}
A(x)= & \frac{\frac{1}{\Gamma(\alpha)} \int_{0}^{T}\left(\int_{0}^{t}(t-s)^{\alpha-1} g(t) h(s) d s\right) d t+y(x) \int_{0}^{T} g(t) d t}{1-\frac{1}{T} \int_{0}^{T} \operatorname{tg}(t) d t} \\
& -\frac{\frac{1}{T \Gamma(\alpha)} \int_{0}^{T}\left(\int_{0}^{T}(T-s)^{\alpha-1} \operatorname{tg}(t) h(s) d s\right) d t+\frac{y(x)}{T} \int_{0}^{T} \operatorname{tg}(t) d t}{1-\frac{1}{T} \int_{0}^{T} \operatorname{tg}(t) d t} .
\end{aligned}
$$

Proof By applying Lemma 2.2, we may reduce (2.4) to an equivalent integral equation

$$
x(t)=\frac{1}{\Gamma(\alpha)} \int_{0}^{t}(t-s)^{\alpha-1} h(s) d s+c_{0}+c_{1} t
$$

for some $c_{0}, c_{1} \in \mathbb{R}$. From (2.5), it follows

$$
c_{0}=y(x), \quad c_{1}=\frac{1}{T} \int_{0}^{T} g(s) x(s) d s-\frac{1}{T \Gamma(\alpha)} \int_{0}^{T}(T-s)^{\alpha-1} h(s) d s-\frac{1}{T} y(x) .
$$

Let $A(x)=\int_{0}^{T} g(s) x(s) d s$. Then

$$
\begin{aligned}
A(x)= & \frac{\frac{1}{\Gamma(\alpha)} \int_{0}^{T}\left(\int_{0}^{t}(t-s)^{\alpha-1} g(t) h(s) d s\right) d t+y(x) \int_{0}^{T} g(t) d t}{1-\frac{1}{T} \int_{0}^{T} \operatorname{tg}(t) d t} \\
& -\frac{\frac{1}{T \Gamma(\alpha)} \int_{0}^{T}\left(\int_{0}^{T}(T-s)^{\alpha-1} \operatorname{tg}(t) h(s) d s\right) d t+\frac{y(x)}{T} \int_{0}^{T} \operatorname{tg}(t) d t}{1-\frac{1}{T} \int_{0}^{T} \operatorname{tg}(t) d t},
\end{aligned}
$$

and so

$$
\begin{aligned}
x(t)= & \frac{1}{\Gamma(\alpha)} \int_{0}^{t}(t-s)^{\alpha-1} h(s) d s+y(x)+\frac{t}{T} A(x) \\
& -\frac{t}{T \Gamma(\alpha)} \int_{0}^{T}(T-s)^{\alpha-1} h(s) d s-\frac{t}{T} y(x) .
\end{aligned}
$$

\section{Main results}

Now we are in a position to establish the main results. First, we are going to deal with problems (1.1) and (1.2).

\section{Theorem 3.1 Assume that:}

(H1) There exists a constant $k>0$ such that $\left|f(t, u)-f\left(t, u_{1}\right)\right| \leq k\left|u-u_{1}\right|$ for each $t \in[0, T]$ and all $u, u_{1} \in \mathbb{R}$.

(H2) There exists a constant $k_{1}>0$ such that $\left|y(u)-y\left(u_{1}\right)\right| \leq k_{1}\left|u-u_{1}\right|$ for each $u, u_{1} \in C^{2}([0, T], \mathbb{R})$.

(H3) $\left(\frac{T^{\alpha}}{\Gamma(\alpha+1)}+\frac{2 T^{\alpha}}{\Gamma(\alpha+2)}\right) k+k_{1}<1$.

Then boundary value problem (1.1)-(1.2) has a unique solution. 
Proof Transform boundary value problem (1.1)-(1.2) into a fixed point problem. For this purpose, we consider the operator

$$
F: C^{2}([0, T], \mathbb{R}) \rightarrow C^{2}([0, T], \mathbb{R})
$$

defined by

$$
\begin{aligned}
F(x)(t)= & \frac{1}{\Gamma(\alpha)} \int_{0}^{t}(t-s)^{\alpha-1} f(s, x(s)) d s+\frac{2 m}{T^{2}} t+\left(\frac{T-2 t}{T}\right) y(x) \\
& -\frac{2 t}{T^{2}} \cdot \frac{1}{\Gamma(\alpha+1)} \int_{0}^{T}(T-s)^{\alpha} f(s, x(s)) d s .
\end{aligned}
$$

Clearly, the fixed points of the operator $F$ are solutions of problem (1.1)-(1.2). Let $x, x_{1} \in$ $C^{2}([0, T], \mathbb{R})$. For each $t \in[0, T]$, we have

$$
\begin{aligned}
&\left|F(x)(t)-F\left(x_{1}\right)(t)\right| \\
& \leq \frac{1}{\Gamma(\alpha)} \int_{0}^{t}(t-s)^{\alpha-1}\left|f(s, x(s))-f\left(s, x_{1}(s)\right)\right| d s+\left|\frac{T-2 t}{T}\right|\left|y(x)-y\left(x_{1}\right)\right| \\
&+\frac{2 t}{T^{2} \Gamma(\alpha+1)} \int_{0}^{T}(T-s)^{\alpha}\left|f(s, x(s))-f\left(s, x_{1}(s)\right)\right| d s \\
& \leq \frac{k\left\|x-x_{1}\right\|}{\Gamma(\alpha)} \cdot \frac{t^{\alpha}}{\alpha}+k_{1}\left\|x-x_{1}\right\|+\frac{2}{T \Gamma(\alpha+1)} k\left\|x-x_{1}\right\| \cdot \frac{T^{\alpha+1}}{\alpha+1} \\
& \leq {\left[\left(\frac{T^{\alpha}}{\Gamma(\alpha+1)}+\frac{2 T^{\alpha}}{\Gamma(\alpha+2)}\right) k+k_{1}\right]\left\|x-x_{1}\right\| . }
\end{aligned}
$$

Thus

$$
\left\|F(x)(t)-F\left(x_{1}\right)(t)\right\| \leq\left[\left(\frac{T^{\alpha}}{\Gamma(\alpha+1)}+\frac{2 T^{\alpha}}{\Gamma(\alpha+2)}\right) k+k_{1}\right]\left\|x-x_{1}\right\| .
$$

Consequently, $F$ is a contraction. As a consequence of Banach's fixed point theorem, we deduce that $F$ has a fixed point which is the solution of problem (1.1)-(1.2).

\section{Theorem 3.2 Assume that:}

(H3) The function $f:[0, T] \times \mathbb{R} \rightarrow \mathbb{R}$ is $C^{2}$ continuous.

(H4) There exists a constant $M>0$ such that $|f(t, u)| \leq M$ for each $t \in[0, T]$ and $u \in \mathbb{R}$.

(H5) There exists a constant $M_{1}>0$ such that $|y(x)| \leq M_{1}$ for each $x \in C^{2}([0, T], \mathbb{R})$.

Then boundary value problem (1.1)-(1.2) has at least one solution.

Proof We will use Schaefer's fixed point theorem to prove this result. We divide the proof into four steps.

(a) First we show that $F$ is continuous. Let $x_{n}$ be a sequence such that $x_{n} \rightarrow x$ in $C^{2}([0, T], \mathbb{R})$. Then, for each $t \in[0, T]$,

$$
\begin{aligned}
\left|F\left(x_{n}\right)(t)-F(x)(t)\right| \leq & \frac{1}{\Gamma(\alpha)} \int_{0}^{t}(t-s)^{\alpha-1}\left|f\left(s, x_{n}(s)\right)-f(s, x(s))\right| d s+\left|y\left(x_{n}\right)-y(x)\right| \\
& +\frac{2}{T \Gamma(\alpha+1)} \int_{0}^{T}(T-s)^{\alpha}\left|f\left(s, x_{n}(s)\right)-f(s, x(s))\right| d s .
\end{aligned}
$$


Since $f$ and $y$ are continuous functions, we have

$$
\left\|F\left(x_{n}\right)(t)-F(x)(t)\right\| \rightarrow 0 \quad \text { as } n \rightarrow \infty
$$

This means that $F$ is continuous.

(b) Next we prove that $F$ maps bounded sets into bounded sets in $C^{2}([0, T], \mathbb{R})$. Indeed, for each $t \in[0, T]$, we have

$$
|F(x)(t)| \leq\left(\frac{T^{\alpha}}{\Gamma(\alpha+1)}+\frac{2 T^{\alpha}}{\Gamma(\alpha+2)}\right) M+M_{1}+\frac{2}{T}|m| .
$$

Thus $F$ is uniformly bounded.

(c) Now we verify that $F$ maps bounded sets into equicontinuous sets of $C^{2}([0, T], \mathbb{R})$.

For each $t_{1}, t_{2} \in[0, T], t_{1}<t_{2}$, we have

$$
\begin{aligned}
\left|F(x)\left(t_{2}\right)-F(x)\left(t_{1}\right)\right| \\
\leq \mid \frac{1}{\Gamma(\alpha)} \int_{0}^{t_{1}}\left[\left(t_{2}-s\right)^{\alpha-1}-\left(t_{1}-s\right)^{\alpha-1}\right] f(s, x(s)) d s \\
\quad+\frac{1}{\Gamma(\alpha)} \int_{t_{1}}^{t_{2}}\left(t_{2}-s\right)^{\alpha-1} f(s, x(s)) d s\left|+\frac{2|m|}{T^{2}}\left(t_{2}-t_{1}\right)+\frac{2\left(t_{2}-t_{1}\right)}{T}\right| y(x) \mid \\
\quad+\left|\frac{2 t_{2}}{T^{2} \Gamma(\alpha+1)}-\frac{2 t_{1}}{T^{2} \Gamma(\alpha+1)}\right| \cdot\left|\int_{0}^{T}(T-s)^{\alpha} f(s, x(s)) d s\right| \\
\leq \frac{M}{\Gamma(\alpha)} \int_{0}^{t_{1}}\left[\left(t_{2}-s\right)^{\alpha-1}-\left(t_{1}-s\right)^{\alpha-1}\right] d s+\frac{M}{\Gamma(\alpha)} \int_{t_{1}}^{t_{2}}\left(t_{2}-s\right)^{\alpha-1} d s \\
\quad+\frac{2|m|}{T^{2}}\left(t_{2}-t_{1}\right)+\frac{2 M_{1}}{T}\left(t_{2}-t_{1}\right)+\frac{2\left(t_{2}-t_{1}\right)}{T^{2} \Gamma(\alpha+1)} \frac{T^{\alpha+1} M}{\alpha+1} \\
=\frac{M}{\Gamma(\alpha+1)}\left(t_{2}^{\alpha}-t_{1}^{\alpha}\right)+\frac{2|m|}{T^{2}}\left(t_{2}-t_{1}\right)+\frac{2 M_{1}}{T}\left(t_{2}-t_{1}\right)+\frac{2 M T^{\alpha-1}}{\Gamma(\alpha+2)}\left(t_{2}-t_{1}\right),
\end{aligned}
$$

which implies that if $t_{1} \rightarrow t_{2}$, the right-hand side of the above inequality tends to zero.

As a consequence of the first three steps above, together with the Arzela-Ascoli theorem, we get that $F$ is completely continuous.

(d) Now it remains to show that the set $E=\left\{x \in C^{2}([0, T], \mathbb{R}): X=\lambda F(x), 0<\lambda<1\right\}$ is bounded. Let $x \in E$, then $x=\lambda F(x), 0<\lambda<1$. Thus, for each $t \in[0, T]$, we have

$$
\begin{aligned}
x= & \lambda F(x)(t) \\
= & \frac{\lambda}{\Gamma(\alpha)} \int_{0}^{t}(t-s)^{\alpha-1} f(s, x(s)) d s+\frac{2 \lambda m}{T^{2}} t+\lambda\left(\frac{T-2 t}{T}\right) y(x) \\
& -\frac{2 t}{T^{2}} \cdot \frac{\lambda}{\Gamma(\alpha+1)} \int_{0}^{T}(T-s)^{\alpha} f(s, x(s)) d s .
\end{aligned}
$$

This implies by (3.1) that for each $t \in[0, T]$, we have

$$
\|x\| \leq\left(\frac{T^{\alpha}}{\Gamma(\alpha+1)}+\frac{2 T^{\alpha}}{\Gamma(\alpha+2)}\right) M+M_{1}+\frac{2}{T}|m| .
$$


This shows that the set $E$ is bounded. As a consequence of Schaefer's fixed point theorem, we deduce that $F$ has a fixed point which is a solution of boundary value problem (1.1)(1.2). The proof is completed.

In the following, we give the existence and uniqueness of a solution for problems (1.1) and (1.3).

Theorem 3.3 Assume that:

(H6) There exists a constant $k>0$ such that $\left|f(t, u)-f\left(t, u_{1}\right)\right| \leq k\left|u-u_{1}\right|$ for each $t \in[0, T]$ and all $u, u_{1} \in \mathbb{R}$.

(H7) There exists a constant $k_{1}>0$ such that $\left|y(u)-y\left(u_{1}\right)\right| \leq k_{1}\left|u-u_{1}\right|$ for each $u, u_{1} \in C^{2}([0, T], \mathbb{R})$.

(H8) $0<g(t)<M$ and $1-\frac{1}{2} M T>0$ for each $t \in[0, T]$.

(H9) $\left(\frac{2 T^{\alpha}}{\Gamma(\alpha+1)}+\frac{T^{\alpha+1} M}{\Gamma(\alpha+1)(2-M T)}+\frac{2 T^{\alpha+1} M}{\Gamma(\alpha+2)(2-M T)}\right) k+\frac{2+2 M T}{2-M T} k_{1}<1$.

Then boundary value problem (1.1)-(1.3) has a unique solution.

Proof Consider the operator

$$
F: C^{2}([0, T], \mathbb{R}) \rightarrow C^{2}([0, T], \mathbb{R})
$$

defined by

$$
\begin{aligned}
F(x)(t)= & \frac{1}{\Gamma(\alpha)} \int_{0}^{t}(t-s)^{\alpha-1} f(s, x(s)) d s+y(x)+\frac{t}{T} A(x) \\
& -\frac{t}{T \Gamma(\alpha)} \int_{0}^{T}(T-s)^{\alpha-1} f(s, x(s)) d s-\frac{t}{T} y(x),
\end{aligned}
$$

where

$$
\begin{aligned}
A(x)= & \frac{\frac{1}{\Gamma(\alpha)} \int_{0}^{T}\left(\int_{0}^{t}(t-s)^{\alpha-1} g(t) f(s, x(s)) d s\right) d t+y(x) \int_{0}^{T} g(t) d t}{1-\frac{1}{T} \int_{0}^{T} \operatorname{tg}(t) d t} \\
& -\frac{\frac{1}{T \Gamma(\alpha)} \int_{0}^{T}\left(\int_{0}^{T}(T-s)^{\alpha-1} \operatorname{tg}(t) f(s, x(s)) d s\right) d t+\frac{y(x)}{T} \int_{0}^{T} \operatorname{tg}(t) d t}{1-\frac{1}{T} \int_{0}^{T} \operatorname{tg}(t) d t} .
\end{aligned}
$$

Let $x, x_{1} \in C^{2}([0, T], \mathbb{R})$ for each $t \in[0, T]$. Then we have

$$
\begin{aligned}
\left|F(x)(t)-F\left(x_{1}\right)(t)\right| & \\
\leq & \frac{1}{\Gamma(\alpha)} \int_{0}^{t}(t-s)^{\alpha-1}\left|f(s, x(s))-f\left(s, x_{1}(s)\right)\right| d s+\left|\frac{T-t}{T}\right|\left|y(x)-y\left(x_{1}\right)\right| \\
& +\frac{t}{T}\left|A(x)-A\left(x_{1}\right)\right|+\frac{t}{T \Gamma(\alpha)} \int_{0}^{T}(T-s)^{\alpha-1}\left|f(s, x(s))-f\left(s, x_{1}(s)\right)\right| d s \\
\leq & \frac{2 T^{\alpha} k\left\|x-x_{1}\right\|}{\Gamma(\alpha+1)}+\frac{\frac{2}{\Gamma(\alpha+2)}+\frac{1}{\Gamma(\alpha+1)}}{2-M T} T^{\alpha+1} M k\left\|x-x_{1}\right\|+\frac{2 k_{1}+2 M T k_{1}}{2-M T}\left\|x-x_{1}\right\|,
\end{aligned}
$$

where $A\left(x_{1}\right)$ means that we use $x_{1}$ to replace $x$ in $A(x)$. 
Thus

$$
\begin{aligned}
\left\|F(x)(t)-F\left(x_{1}\right)(t)\right\| \leq & {\left[\left(\frac{2 T^{\alpha}}{\Gamma(\alpha+1)}+\frac{T^{\alpha+1} M}{\Gamma(\alpha+1)(2-M T)}+\frac{2 T^{\alpha+1} M}{\Gamma(\alpha+2)(2-M T)}\right) k\right.} \\
& \left.+\frac{2+2 M T}{2-M T} k_{1}\right]\left\|x-x_{1}\right\| .
\end{aligned}
$$

Consequently, $F$ is a contraction. Therefore, the fixed point theorem implies that boundary value problem (1.1)-(1.3) has a unique solution in $C^{2}([0, T], \mathbb{R})$. And the proof is completed.

Theorem 3.4 Assume that:

(H10) The function $f:[0, T] \times \mathbb{R} \rightarrow \mathbb{R}$ is $C^{2}$ continuous.

(H11) There exists a constant $M_{1}>0$ such that $|f(t, u)| \leq M_{1}$ for each $t \in[0, T]$ and $u \in \mathbb{R}$.

(H12) There exists a constant $M_{2}>0$ such that $|y(x)| \leq M_{2}$ for each $x \in C^{2}([0, T], \mathbb{R})$.

(H13) $0<g(t)<M$ and $1-\frac{1}{2} M T>0$ for each $t \in[0, T]$.

Then boundary value problem (1.1)-(1.3) has at least one solution.

Proof Similar to the proof of Theorem 3.2, we will prove the theorem by showing the following four steps.

(a) Show that $F$ is continuous. Let $x_{n}$ be a sequence such that $x_{n} \rightarrow x$ in $C^{2}([0, T], \mathbb{R})$. Then for each $t \in[0, T]$,

$$
\begin{aligned}
& \left|F\left(x_{n}\right)(t)-F(x)(t)\right| \\
& \leq \leq \frac{1}{\Gamma(\alpha)} \int_{0}^{t}(t-s)^{\alpha-1}\left|f\left(s, x_{n}(s)\right)-f(s, x(s))\right| d s+\left|y\left(x_{n}\right)-y(x)\right| \\
& \quad+\frac{1}{\Gamma(\alpha)} \int_{0}^{T}(T-s)^{\alpha-1}\left|f\left(s, x_{n}(s)\right)-f(s, x(s))\right| d s+\frac{1.5 M T\left|y\left(x_{n}\right)-y(x)\right|}{1-0.5 M T} \\
& \quad+\frac{\frac{M}{\Gamma(\alpha)} \int_{0}^{T}\left(\int_{0}^{t}(t-s)^{\alpha-1}\left|f\left(s, x_{n}(s)\right)-f(s, x(s))\right| d s\right) d t}{1-0.5 M T} \\
& \quad+\frac{\frac{M}{\Gamma(\alpha)} \int_{0}^{T}\left(\int_{0}^{T}(T-s)^{\alpha-1}\left|f\left(s, x_{n}(s)\right)-f(s, x(s))\right| d s\right) d t}{1-0.5 M T} .
\end{aligned}
$$

Since $f$ and $y$ are continuous functions, then we have

$$
\left\|F\left(x_{n}\right)(t)-F(x)(t)\right\| \rightarrow 0 \quad \text { as } n \rightarrow \infty
$$

This means that $F$ is continuous.

(b) Verify $F$ maps bounded sets into bounded sets in $C^{2}([0, T], \mathbb{R})$. Indeed, for each $t \in$ $[0, T]$, we have

$$
|F(x)(t)| \leq \frac{2 M_{1} T^{\alpha}}{\Gamma(\alpha+1)}+M_{2}+\frac{\frac{M M_{1} T^{\alpha+1}}{\Gamma(\alpha+2)}+1.5 M M_{2} T+\frac{M M_{1} T^{\alpha+1}}{2 \Gamma(\alpha+1)}}{1-0.5 M T}
$$

Thus $F$ is uniform bounded. 
(c) Examine $F$ maps bounded sets into equicontinuous sets of $C^{2}([0, T], \mathbb{R})$. For each $t_{1}, t_{2} \in[0, T], t_{1}<t_{2}$, we have

$$
\begin{aligned}
\left|F(x)\left(t_{2}\right)-F(x)\left(t_{1}\right)\right| \leq & \frac{1}{\Gamma(\alpha)} \int_{0}^{t_{1}}\left[\left(t_{2}-s\right)^{\alpha-1}-\left(t_{1}-s\right)^{\alpha-1}\right] f(s, x(s)) d s \\
& +\frac{1}{\Gamma(\alpha)} \int_{t_{1}}^{t_{2}}\left(t_{2}-s\right)^{\alpha-1} f(s, x(s)) d s\left|+\frac{\left(t_{2}-t_{1}\right)}{T}\right| y(x) \mid \\
& +\frac{\left(t_{2}-t_{1}\right)}{T \Gamma(\alpha)}\left|\int_{0}^{T}(T-s)^{\alpha-1} f(s, x(s)) d s\right|+\left(\frac{t_{2}}{T}-\frac{t_{1}}{T}\right)|A(x)| \\
\leq & \frac{M_{1}}{\Gamma(\alpha+1)}\left(t_{2}^{\alpha}-t_{1}^{\alpha}\right)+\frac{M_{2}}{T}\left(t_{2}-t_{1}\right)+\frac{M_{1} T^{\alpha}}{T \Gamma(\alpha+1)}\left(t_{2}-t_{1}\right) \\
& +\frac{\frac{M M_{1} T^{\alpha+1}}{\Gamma(\alpha+2)}+1.5 M M_{2} T+\frac{M M_{1} T^{\alpha+1}}{2 \Gamma(\alpha+1)}}{1-0.5 M T} \frac{\left(t_{2}-t_{1}\right)}{T} .
\end{aligned}
$$

Hence the right-hand side of the above inequality tends to zero as $t_{1} \rightarrow t_{2}$.

As a consequence of (a) to (c) together with the Arzela-Ascoli theorem, we get that $F$ : $C^{2}([0, T], \mathbb{R}) \rightarrow C^{2}([0, T], \mathbb{R})$ is completely continuous.

(d) In what follows, we will show that the set $E=\left\{x \in C^{2}([0, T], \mathbb{R}): x=\lambda F(x), 0<\lambda<1\right\}$ is bounded. Let $x \in E$. Then $x=\lambda F(x), 0<\lambda<1$, and for each $t \in[0, T]$, we have

$$
\begin{aligned}
x= & \lambda F(x)(t) \\
= & \frac{\lambda}{\Gamma(\alpha)} \int_{0}^{t}(t-s)^{\alpha-1} f(s, x(s)) d s+\lambda y(x)+\frac{\lambda t}{T} A(x) \\
& -\frac{\lambda t}{T \Gamma(\alpha)} \int_{0}^{T}(T-s)^{\alpha-1} f(s, x(s)) d s-\frac{\lambda t}{T} y(x),
\end{aligned}
$$

which implies by (3.2) that for each $t \in[0, T]$, we have

$$
\|x\| \leq \frac{2 M_{1} T^{\alpha}}{\Gamma(\alpha+1)}+M_{2}+\frac{\frac{M M_{1} T^{\alpha+1}}{\Gamma(\alpha+2)}+1.5 M M_{2} T+\frac{M M_{1} T^{\alpha+1}}{2 \Gamma(\alpha+1)}}{1-0.5 M T} .
$$

This shows that the set $E$ is bounded. As a consequence of Schaefer's fixed point theorem, we deduce that $F$ has a fixed point which is a solution of boundary value problem (1.1)-(1.3).

\section{Examples}

In this section, we give some examples to illustrate our main results.

Example 4.1 Consider

$$
\begin{aligned}
& D_{0+}^{1.5} x(t)=\frac{e^{-t}}{9+e^{t}} \frac{|x|}{1+|x|}, \\
& x(0)=\sum_{i=1}^{n} c_{i} x\left(t_{i}\right), \quad \int_{0}^{1} x(t) d t=1,
\end{aligned}
$$

where $0<t_{1}<t_{2}<\cdots<t_{n}<1, c_{i}, i=1,2, \ldots, n$, are given positive constants with $\sum_{i=1}^{n} c_{i}<\frac{1}{2}$. Consider boundary value problem (1.1)-(1.2) with $\alpha=1.5, f(t, x(t))=\frac{e^{-t}}{9+e^{t}} \frac{|x|}{1+|x|}, y(x)=$ $\sum_{i=1}^{n} c_{i} x\left(t_{i}\right), m=1, T=1$. 
Let $t \in[0.1]$ and $u, u_{1} \in \mathbb{R}$. Then

$$
\left|f(t, u)-f\left(t, u_{1}\right)\right|=\left|\frac{e^{-t}}{9+e^{t}}\right|\left|\frac{|x|}{1+|x|}\right| \leq \frac{e^{-t}}{9+e^{t}}|| u|-| u_{1}|| \leq \frac{1}{10}\left|u-u_{1}\right| .
$$

Hence the condition (H1) holds with $k=\frac{1}{10}$. Also, we have

$$
\left|y(u)-y\left(u_{1}\right)\right|=\left|\sum_{i=1}^{n} c_{i} u\left(t_{i}\right)-\sum_{i=1}^{n} c_{i} u_{1}\left(t_{i}\right)\right| \leq \sum_{i=1}^{n}\left|u-u_{1}\right| .
$$

So, (H2) is satisfied with $k_{1}=\sum_{i=1}^{n} c_{i}<\frac{1}{2}$.

Therefore, we can rest easy knowing that

$$
\left(\frac{T^{\alpha}}{\Gamma(\alpha+1)}+\frac{2 T^{\alpha}}{\Gamma(\alpha+2)}\right) k+k_{1}=\left(\frac{1}{\Gamma(2.5)}+\frac{2}{\Gamma(3.5)}\right) \cdot \frac{1}{10}+\sum_{i=1}^{n} c_{i}<1
$$

Thus, by Theorem 3.1, boundary value problem (4.1)-(4.2) has a unique solution.

Example 4.2 Consider

$$
\begin{aligned}
& D_{0+}^{1.5} x(t)=\frac{t^{\frac{1}{3}} e^{-2 t}}{1+t x^{2}}, \\
& x(0)=\sum_{i=1}^{n} c_{i} \frac{\left|x\left(t_{i}\right)\right|}{1+\left|x\left(t_{i}\right)\right|}, \quad \int_{0}^{1} x(t) d t=1,
\end{aligned}
$$

where $0<t_{1}<t_{2}<\cdots<t_{n}<1, c_{i}, i=1,2, \ldots, n$, are given positive constants with $\sum_{i=1}^{n} c_{i}<\frac{1}{2}$. Consider boundary value problem (1.1)-(1.2) with $\alpha=1.5, f(t, x(t))=\frac{t^{\frac{1}{3}} e^{-2 t}}{1+t x^{2}}, y(x)=$ $\sum_{i=1}^{n} c_{i} \frac{\left|x\left(t_{i}\right)\right|}{1+\left|x\left(t_{i}\right)\right|}, m=1, T=1$.

Clearly,

$$
|f(t, x)| \leq\left|\frac{t^{\frac{1}{3}} e^{-2 t}}{1+t x^{2}}\right| \leq 1=M, \quad|y(x)| \leq \sum_{i=1}^{n} c_{i} \frac{\left|x\left(t_{i}\right)\right|}{1+\left|x\left(t_{i}\right)\right|} \leq \frac{1}{2}=M_{1}
$$

Hence, all the conditions of Theorem 3.2 are satisfied and consequently boundary value problem (4.3)-(4.4) has at least one solution.

Example 4.3 Consider

$$
\begin{aligned}
& D_{0+}^{1.5} x(t)=\frac{e^{-t}}{9+e^{t}} \frac{|x|}{1+|x|}, \\
& x(0)=\sum_{i=1}^{n} c_{i} x\left(t_{i}\right), \quad x(1)=\int_{0}^{1} x(t) \sin t d t,
\end{aligned}
$$

where $0<t_{1}<t_{2}<\cdots<t_{n}<1, c_{i}, i=1,2, \ldots, n$, are given positive constants with $\sum_{i=1}^{n} c_{i}<\frac{1}{8}$. Consider boundary value problem (1.1)-(1.3) with $\alpha=1.5, f(t, x(t))=\frac{e^{-t}}{9+e^{t}} \frac{|x|}{1+|x|}, y(x)=$ $\sum_{i=1}^{n} c_{i} x\left(t_{i}\right), g(t)=\sin t, T=1$. 
Let $t \in[0,1]$ and $u, u_{1} \in \mathbb{R}$. Then

$$
\left|f(t, u)-f\left(t, u_{1}\right)\right|=\left|\frac{e^{-t}}{9+e^{t}}\right|\left|\frac{|x|}{1+|x|}\right| \leq \frac{e^{-t}}{9+e^{t}}|| u|-| u_{1}|| \leq \frac{1}{10}\left|u-u_{1}\right| .
$$

Hence the condition (H6) holds with $k=\frac{1}{10}$. Also, we have

$$
\left|y(u)-y\left(u_{1}\right)\right|=\left|\sum_{i=1}^{n} c_{i} u\left(t_{i}\right)-\sum_{i=1}^{n} c_{i} u_{1}\left(t_{i}\right)\right| \leq \sum_{i=1}^{n} c_{i}\left|u-u_{1}\right| .
$$

Hence (H7) is satisfied with $k_{1}=\sum_{i=1}^{n} c_{i}<\frac{1}{8}$. Set $M=1$. Then (H8) is satisfied.

We can show that

$$
\left(\frac{2 T^{\alpha}}{\Gamma(\alpha+1)}+\frac{T^{\alpha+1} M}{\Gamma(\alpha+1)(2-M T)}+\frac{2 T^{\alpha+1} M}{\Gamma(\alpha+2)(2-M T)}\right) k+\frac{2+2 M T}{2-M T} k_{1} \simeq 0.618<1 .
$$

Then, by Theorem 3.3, boundary value problem (4.5)-(4.6) has a unique solution.

Example 4.4 Consider

$$
\begin{aligned}
& D_{0+}^{1.5} x(t)=\frac{t^{\frac{1}{3}} e^{-2 t}}{1+t x^{2}}, \\
& x(0)=\sum_{i=1}^{n} c_{i} \frac{\left|x\left(t_{i}\right)\right|}{1+\left|x\left(t_{i}\right)\right|}, \quad x(1)=\int_{0}^{1} x(t) \sin t d t,
\end{aligned}
$$

where $0<t_{1}<t_{2}<\cdots<t_{n}<1, c_{i}, i=1,2, \ldots, n$, are given positive constants with $\sum_{i=1}^{n} c_{i}<\frac{1}{2}$. Consider boundary value problem (1.1)-(1.3) with $\alpha=1.5, f(t, x(t))=\frac{t^{\frac{1}{3}} e^{-2 t}}{1+t x^{2}}, y(x)=$ $\sum_{i=1}^{n} c_{i} \frac{\left|x\left(t_{i}\right)\right|}{1+\left|x\left(t_{i}\right)\right|}, g(t)=\sin t, T=1$.

Clearly,

$$
\begin{aligned}
& |f(t, x)| \leq\left|\frac{t^{\frac{1}{3}} e^{-2 t}}{1+t x^{2}}\right| \leq 1=M_{1}, \\
& |y(x)| \leq \sum_{i=1}^{n} c_{i} \frac{\left|x\left(t_{i}\right)\right|}{1+\left|x\left(t_{i}\right)\right|} \leq \frac{1}{2}=M_{2}, \quad 0<g(t)<1=M .
\end{aligned}
$$

Hence, all the conditions of Theorem 3.4 are satisfied, and consequently boundary value problem (4.7)-(4.8) has at least one solution.

\section{Conclusion}

This paper studies the existence and uniqueness of solutions for the fractional differential equations with one nonlocal and one integral boundary conditions, and some results are given by using Banach's fixed point theorem and Schaefer's fixed point theorem. At the foundation of this paper, one can consider boundary value problems of fractional differential equations with parameters, and also can make further research on eigenvalue problems of fractional differential equations. 


\section{Authors' contributions}

The authors declare that the study was realized in collaboration with the same responsibility. All authors read and approved the final manuscript.

\section{Acknowledgements}

The authors sincerely thank the reviewers for their valuable suggestions and useful comments that have led to the present improved version of the original manuscript. This research is supported by the Natural Science Foundation of China (11071143), Natural Science Outstanding Youth Foundation of Shandong Province (JQ201119) and supported by Shandong Provincial Natural Science Foundation (ZR2012AM009, ZR2011 AL007), also supported by Natural Science Foundation of Educational Department of Shandong Province (J11LA01).

\section{Received: 26 November 2012 Accepted: 29 May 2013 Published: 18 June 2013}

\section{References}

1. Oldham, KB, Spanier, J: The Fractional Calculus. Academic Press, New York (1974)

2. Kilbas, AA, Srivastava, HH, Trujillo, JJ: Theory and Applications of Fractional Differential Equations. Elsevier, Amsterdam (2006)

3. Zhang, S: The existence of a positive solution for nonlinear fractional differential equation. J. Math. Anal. Appl. 252, 804-812 (2000)

4. Zhang, S: Existence of positive solutions for some class of nonlinear fractional equation. J. Math. Anal. Appl. 278, 136-148 (2003)

5. Zhou, Y, Jiao, F, Li, J: Existence and uniqueness for $p$-type fractional neutral differential equations. Nonlinear Anal. 71, 2724-2733 (2009)

6. Sun, S, Li, Q, Li, Y: Existence and uniqueness of solutions for a coupled system of multi-term nonlinear fractional differential equations. Comput. Math. Appl. 64, 3310-3320 (2012)

7. Zhao, Y, Sun, S, Han, Z, Zhang, M: Positive solutions for boundary value problems of nonlinear fractional differential equations. Appl. Math. Comput. 217, 6950-6958 (2011)

8. Zhao, Y, Sun, S, Han, Z, Li, Q: Positive solutions to boundary value problems of nonlinear fractional differential equations. Abstr. Appl. Anal. 2011, Article ID 390543 (2011). doi:10.1155/2011/390543

9. Zhao, Y, Sun, S, Han, Z, Li, Q: The existence of multiple positive solutions for boundary value problems of nonlinear fractional differential equations. Commun. Nonlinear Sci. Numer. Simul. 16, 2086-2097 (2011)

10. Zhao, Y, Sun, S, Han, Z, Li, Q: Theory of fractional hybrid differential equations. Comput. Math. Appl. 62, 1312-1324 (2011)

11. Feng, W, Sun, S, Han, Z, Zhao, Y: Existence of solutions for a singular system of nonlinear fractional differential equations. Comput. Math. Appl. 62, 1370-1378 (2011)

12. Bai, Z, Lü, H: Positive solutions for boundary value problem of nonlinear fractional differential equation. J. Math. Anal. Appl. 311, 495-505 (2005)

13. Zhang, S: Positive solutions for boundary-value problems of nonlinear fractional differential equations. Electron. J. Differ. Equ. 2006, 36 (2006)

14. Sun, $S, Z$ Zhao, Y, Han, Z, Li, Y: The existence of solutions for boundary value problem of fractional hybrid differential equations. Commun. Nonlinear Sci. Numer. Simul. 17, 4961-4967 (2012)

15. Sun, $\mathrm{S}, \mathrm{Zhao}, \mathrm{Y}, \mathrm{Han}, \mathrm{Z}, \mathrm{Xu}, \mathrm{M}$ : Uniqueness of positive solutions for boundary value problems of singular fractional differential equations. Inverse Probl. Sci. Eng. 20, 299-309 (2012)

16. El-Shahed, M, Nieto, JJ: Nontrivial solutions for a nonlinear multi-point boundary value problem of fractional order. Comput. Math. Appl. 59, 3438-3443 (2010)

17. Rehman, M, Khan, RA: Existence and uniqueness of solutions for multi-point boundary value problems for fractional differential equations. Appl. Math. Lett. 23, 1038-1044 (2010)

18. Gallardo, JM: Second order differential operators with integral boundary conditions and generation of semigroups. Rocky Mt. J. Math. 30, 1265-1291 (2000)

19. Karakostas, GL, Tsamatos, P: Multiple positive solutions of some Fredholm integral equations arisen from nonlocal boundary-value problems. Electron. J. Differ. Equ. 2002, 30 (2002)

20. Zhao, J, Wang, P, Ge, W: Existence and nonexistence of positive solutions for a class of third order BVP with integral boundary conditions in Banach spaces. Commun. Nonlinear Sci. Numer. Simul. 16, 402-413 (2011)

21. Wei, Z, Pang, C, Ding, Y: Positive solutions of singular Caputo fractional differential equations with integral boundary conditions. Commun. Nonlinear Sci. Numer. Simul. 17, 3148-3160 (2012)

22. Cabada, A, Wang, G: Positive solutions of nonlinear fractional differential equations with integral boundary value conditions. J. Math. Anal. Appl. 389, 403-411 (2012)

23. Benchohra, M, Hamani, S, Ntouyas, SK: Boundary value problems for differential equations with fractional order and nonlocal conditions. Nonlinear Anal. 71, 2391-2396 (2009)

doi:10.1186/1687-1847-2013-176

Cite this article as: Yan et al.: Boundary value problems for fractional differential equations with nonlocal boundary conditions. Advances in Difference Equations 2013 2013:176. 